OPEN ACCESS

Edited by:

Gang Wu,

Vrije Universiteit Amsterdam,

Netherlands

Reviewed by:

Monica Mattioli-Belmonte,

Marche Polytechnic University, Italy

Zhongru Gou,

Zhejiang University, China

Jianmin Yang.

Fuzhou University, China

Changchun Zhou,

Sichuan University, China

${ }^{*}$ Correspondence:

Kaili Lin

Iklecnu@aliyun.com:

linkaili@tongji.edu.cn

Penglai Wang

wpl0771@qq.com

tThese authors have contributed equally to this work

Specialty section:

This article was submitted to Biomaterials,

a section of the journal Frontiers in Bioengineering and

Biotechnology

Received: 05 August 2020 Accepted: 04 January 2021

Published: 09 February 2021

Citation:

Zhang C, Zhang T, Geng T, Wang X, Lin K and Wang P (2021)

Dental Implants Loaded With

Bioactive Agents Promote Osseointegration in Osteoporosis:

A Review.

Front. Bioeng. Biotechnol. 9:591796.

doi: 10.3389/fbioe.2021.591796

\section{Dental Implants Loaded With Bioactive Agents Promote Osseointegration in Osteoporosis: A Review}

\author{
Cheng Zhang ${ }^{1 \dagger}$, Tianjia Zhang ${ }^{3 \dagger}$, Tengyu Geng ${ }^{2}$, Xudong Wang ${ }^{3}, K^{2}$ ili Lin $^{3 *}$ and \\ Penglai Wang ${ }^{1,2 *}$
}

${ }^{1}$ School of Stomatology, Xuzhou Medical University, Xuzhou, China, ${ }^{2}$ Affiliated Stomatological Hospital of Xuzhou Medical University, Xuzhou, China, ${ }^{3}$ Department of Oral \& Cranio-Maxillofacial Surgery, Shanghai Ninth People's Hospital, Shanghai Jiao Tong University School of Medicine, Shanghai Key Laboratory of Stomatology, Shanghai Research Institute of Stomatology, Shanghai, China

Implant-supported dentures are widely used in patients with defect or loss of dentition because these have higher chewing efficiency and do not damage the adjacent teeth compared with fixed or removable denture. An implant-supported denture carries the risk of failure in some systemic diseases, including osteoporosis, because of a nonideal local microenvironment. Clinically common physical and chemical modifications are used to change the roughness of the implant surface to promote osseointegration, but they have limitations in promoting osteoinduction and inhibiting bone resorption. Recently, many researchers have focused on the study of bioactive modification of implants and have achieved promising results. Herein we have summarized the progress in bioactive modification strategy to promote osseointegration by regulating the local osteoporotic microenvironment.

Keywords: dental implants, bioactive agents, osseointegration, osteoporosis, controlled release

\section{INTRODUCTION}

With innovations in implant design and surgery technology, the applicable conditions of implant surgery have become more extensive, and the 10-year survival rate of an implant-supported denture has exceeded 95\% (Buser et al., 2017). There is a risk of failure in a number of systemic diseases (Liu et al., 2020), including osteoporosis, because the severe decrease in bone mass and alteration of trabecular bone microstructure affect the initial stability and osseointegration of the implants.

Currently, the common sand blasting and acid etching strategy is used to increase the surface roughness of implants, which enhances adhesion, proliferation, and differentiation of mesenchymal stem cells (Zhang et al., 2020; Zhao et al., 2020). In addition to the implant design, it is necessary to promote osteoinduction and inhibit bone resorption locally in osteoporotic patients (Lin et al., 2013). Systemic administration orally or intravenously provides low bioavailability, which makes it difficult to maintain an effective concentration around the implants and might cause severe side effects, such as necrosis of the jaw bone (Eguia et al., 2020). Local administration ensures adequate drug concentration in the target tissue and reduces toxicity in the non-target areas. Hence, it is necessary to biologically modify the implants for loading bioactive agents, such as anti-osteoporosis drugs, bioactive molecules, or bioactive inorganic elements, onto the implant surface. In addition, to avoid burst release in a short time, it is critical to take optimal approaches to optimize the locally 
controlled release of bioactive agents and maintain an effective concentration. Herein we briefly summarize the main progress in this field.

\section{LOADING BIOACTIVE AGENTS TO PROMOTE OSSEOINTEGRATION}

\section{Loading Anti-osteoporosis Drugs}

Anti-osteoporosis drugs are categorized according to their functions and effects and include anti-catabolic drugs, anabolic drugs, and dual-acting drugs (Figure 1). The commonly used anti-catabolic agents for loading on the implant surfaces for local treatment include bisphosphonates, receptor activator of nuclear factor $\mathrm{kB}$ ligand (RANKL) antibodies, and selective estrogen receptor modulators (SERMs), which inhibit the activity and recruitment of osteoclasts or promote the apoptosis of osteoclasts. Bisphosphonates reduce osteoclast activity by inhibiting farnesyl pyrophosphate synthase and protein prenylation in the mevalonate pathway, and SERMs have an agonist effect on the estrogen receptor in osteoclasts, thereby inducing apoptosis (Apostu et al., 2017). Beck et al. (2019) immobilized the RANKL antibody denosumab on the implant surface to inhibit osteoclast differentiation, which could competitively block the interaction between receptor activator of nuclear factor $\mathrm{kB}$ and RANKL.

Being an anabolic drug, the parathyroid hormone (PTH) activates the cyclic adenosine monophosphate/protein kinase A (cAMP/PKA) signaling pathway by acting on the PTH receptors in osteoblasts to promote osteointegration (Tang et al., 2020). PTH 1-34 or teriparatide, a fragment of endogenous PTH, was the main regulator of calcium and phosphate metabolism in bone and the first anabolic drug proven to increase the osseointegration of implants. Other anabolic drugs targeting the Wnt/ $\beta$-catenin signaling pathway have also been studied, such as romosozumab and DKK1 antagonists (Gennari et al., 2020).

Strontium ranelate and simvastatin play dual effects of promoting bone formation and inhibiting bone absorption through various signaling pathways (Apostu et al., 2017). Loading simvastatin and strontium ranelate on the implant surface effectively improves the local bone microenvironment, which is a promising method to enhance the osseointegration of implants in osteoporotic patients (Lai et al., 2018).

\section{Loading Bioactive Molecules}

The term "bioactive agents" is not limited only to therapeutic agents used in the clinic. The scope of this term has been broadened to bioactive molecules, including growth factors, proteins, and genes (Meng et al., 2016; Souza et al., 2019). Although the clinical application of these bioactive molecules is limited because of high production costs and concerns about biosafety, researchers have conducted a lot of research in this area. Loading various growth factors and proteins on the implant surfaces can promote osteogenic differentiation and the mineralization of bone marrow stem cells. Plateletderived growth factor, insulin-like growth factor, fibroblast growth factor, vascular endothelial growth factor (VEGF), and bone morphogenetic protein (BMP) have been widely used in this field (Chen and Zou, 2019; Jurczak et al., 2020). VEGF can promote angiogenesis and regulate bone regeneration; Zavan et al. (2017) loaded VEGF on the surface of the implant in vitro, which effectively enhanced the osteogenic differentiation of stem cells. The release of BMP from the implant surface facilitated the proliferation, differentiation, and mineralization of bone cells in vitro and enhanced bone healing in vivo (Teng et al., 2019).

In addition, the extracellular matrix, such as type I collagen, showed good biological activity and osteoinductivity, which could improve adhesion and differentiation and promote boneto-implant integration (Wang et al., 2019). Similarly, genes can be incorporated into the implant surface to transfect local osteoblasts or osteoclasts around the implants to promote osteoblastogenesis and inhibit osteoclastogenesis. Takanche et al. (2018) performed an experiment in which c-myb, a transcription factor, was delivered from chitosan-gold nanoparticle-coated titanium surface to the target tissue, where it promoted bone formation under osteoporotic conditions.

\section{Modification by Bioactive Inorganic Elements}

Some essential elements, including calcium (Ca), strontium (Sr), magnesium $(\mathrm{Mg})$, zinc $(\mathrm{Zn})$, and silicon $(\mathrm{Si})$, can be also loaded on implants to stimulate osteogenesis (Lin et al., 2013; Liu et al., 2020, 2021). Compared with anti-osteoporosis drugs and bioactive molecules, more efficient strategies can be applied to construct inorganic element coating on the implant surface, which costs less (Asri et al., 2017). Ca is one of the essential micronutrients in bone, and bone structure abnormalities in osteoporotic patients occur as a result of the loss of $\mathrm{Ca}$. At present, Ca-phosphate biomimetic coating on the implant surface is widely used to promote adhesion and differentiation of osteoblasts because of the chemical similarity between the synthetic materials and the bone mineral components (Xia et al., 2018). Sr is often used in the treatment of osteoporosis because of its dual role in bone regulation. An Sr-incorporated implant surface obtained by hydrothermal reaction could promote early osseointegration in osteoporotic rabbits (Lin et al., 2019). Moreover, Mg-immersed titanium-dioxide $\left(\mathrm{TiO}_{2}\right)$ coatings on the implant surface had both osteogenic and antibacterial effects (Zhao et al., 2019). Zn-modified coating on the implant surface facilitated the osteogenic differentiation of bone marrow-derived pericytes through the transforming growth factor-beta/Smad signaling pathway (Yu et al., 2017).

\section{CONTROLLING THE RELEASE BEHAVIORS OF BIOACTIVE AGENTS}

There are many ways to build bioactive organic or inorganic coatings on the implant surface, including physical methods (such as plasma spraying, ion implantation, and physical vapor deposition), chemical methods (such as acid etching and alkali-heat treatment), and electrochemical strategies (such as anodization, micro-arc oxidation, electropolymerization, and electrophoretic deposition) (Asri et al., 2017; Xue et al., 2020). 
Covalent grafting and layer-by-layer self-assembly technology have been used to load bioactive proteins and growth factors. The release of bioactive agents from the implant surface is different because of the different construction methods of the coatings. In practical applications, however, we expect that the drugs loaded on the implants should maintain an effective concentration locally for a long time because burst release not only fails to maintain long-term efficacy but also might cause side effects due to toxicity in local tissues. The controlled release behaviors of the loaded bioactive agents can be achieved mainly by the following means: (1) constructing micro-/nano-structures on the implant surface, (2) introducing a stable immobilization strategy, and (3) encapsulating the bioactive agents.

\section{Constructing Micro-/Nano-structures on the Implant Surface}

Nano- and micro-topography construction on the implant surface not only can provide better biological responses but also may benefit drug adhesion and controlled release (Wang et al., 2018, 2020; Long et al., 2019). The modification of titanium dioxide nanotubes (TNTs) on implant surfaces can significantly increase the surface-to-volume ratio, and this porous surface provides more substantial space for drug loading with better biocompatibility (Figure 2A). More importantly, bioactive agents incorporated on the surface and inside TNTs achieve sustained steady release (Ion et al., 2020). A study conducted by Liu et al. (2018) showed that zoledronate adsorbed on the surface of TNTs can be released steadily for a long time to enhance implant osseointegration. Moreover, the drug release behaviors can be regulated by the diameter and length of TNTs by varying the process conditions of anodic oxidation. Hamlekhan et al. (2015) found that the diameter, length, aspect ratio, and volume were related to the prolonged release process. Moreover, the aspect ratio had the highest correlation with the release rate, and the release process of TNTs with high aspect ratio was significantly slow.

\section{Introducing a Stable Immobilization Strategy}

Bioactive agents can be attached to the implant surface by means of physical adsorption, such as dipping, spray coating, or drop casting, to promote local osseointegration (Alenezi et al., 2019). Physical adsorption characterized by Van der Waals forces or hydrogen bonds, however, leads to burst release at an early stage. Hence, bioactive agents can be covalently immobilized on the surface of titanium indirectly through a separate linker molecule that mediates the binding between titanium substrates and bioactive agents (Figure 2B), which can greatly improve the stability of coatings compared with physical adsorption (Jin et al., 2020). Linker molecules are generally synthetic linkers, such as silane and polyethylene glycol, or biologically derived molecules, such as heparin, dopamine, and chitosan. Linker molecules attach to hydroxyl-functionalized titanium substrates through condensation reactions, and then the bioactive agents are covalently immobilized on the functional group of the linker molecules (Stewart et al., 2019). Covalent bonding is more complex and time consuming than the other techniques, however, and the bioactive agents are not easily released because of the tight covalent bonding (Ma et al., 2020). The layer-by-layer self-assembly technique is being increasingly used for drug loading and controlled release, which can formulate polyelectrolyte multilayers by electrostatic attractions between components with different electric charges (Figure 2C). The selfassembly process is simple and mild, which does not affect the

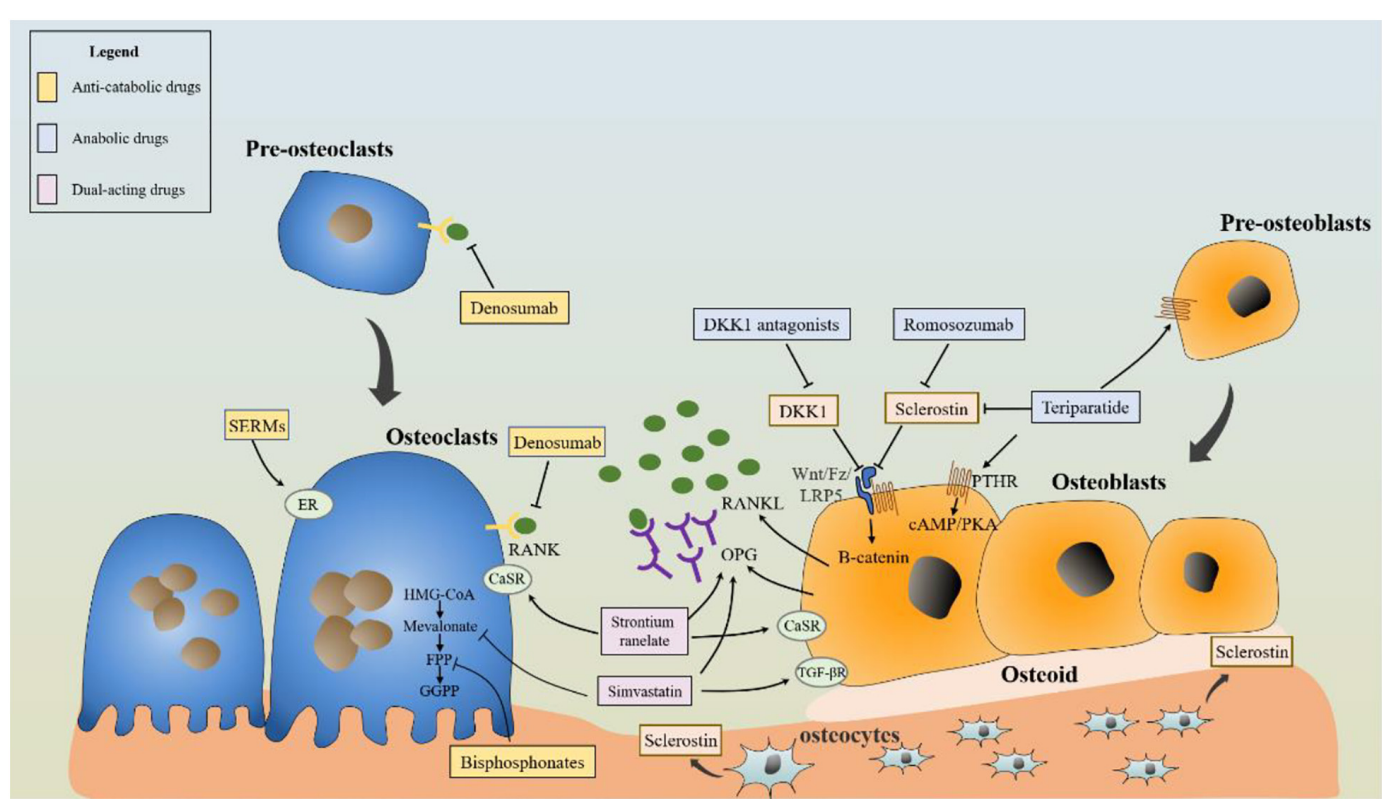

FIGURE 1 | Schematic diagram of anti-osteoporosis drugs acting on osteoblasts and osteoclasts. 

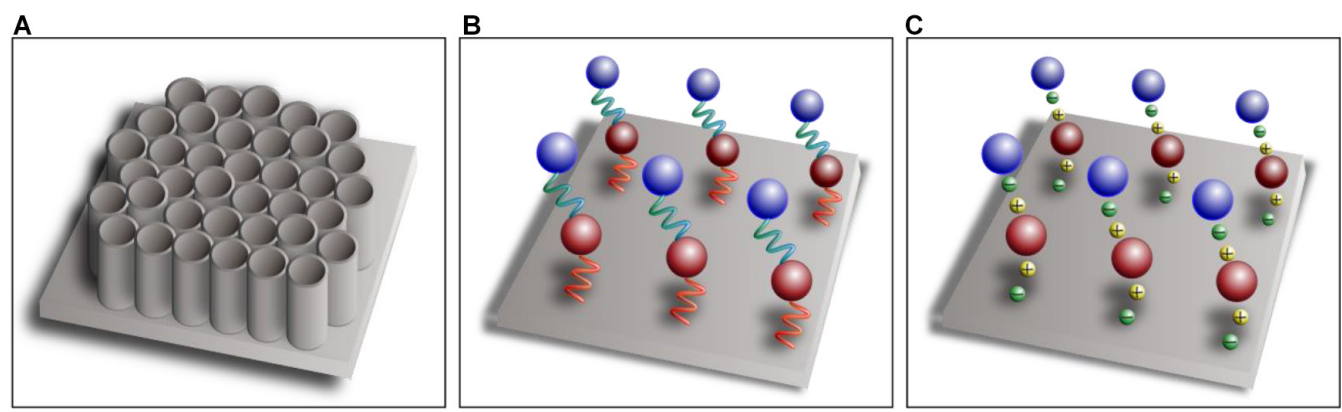

FIGURE 2 | Schematic diagram of titanium dioxide nanotubes (A), covalent immobilization (B), and layer-by-layer self-assembly technique (C).
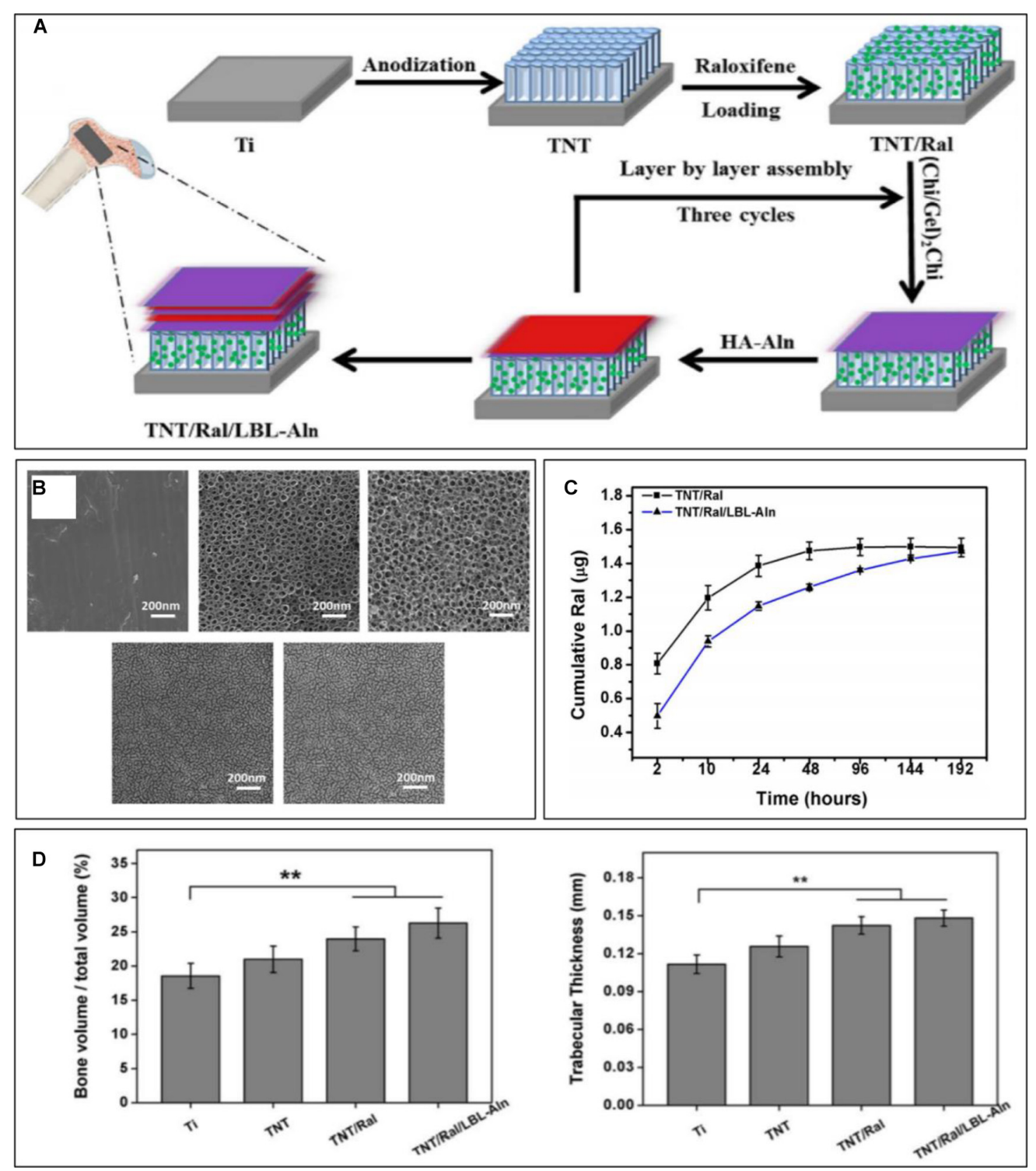

FIGURE 3 | Sustained raloxifene release from $\mathrm{TiO}_{2}$ nanotubes to enhance osteointegration. (A) Schematic illustration of the fabrication of multilayered coating. (B) SEM images of each group. (C) Cumulative release curve of each sample. (D) Quantitative analysis of new bone volume and trabecular thickness [reprinted with

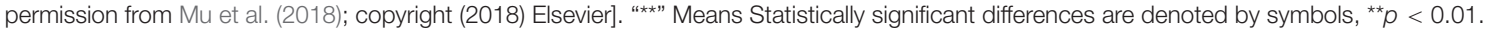


activity of the components, and sustained-release administration can be achieved by adjusting the physical and chemical properties of the materials (Shi et al., 2017).

\section{Encapsulating the Bioactive Agents}

Bioactive agents can be encapsulated by biocompatible materials with appropriate biodegradability, including natural organic polymers [such as chitosan (CS) and gelatin], synthetic organic polymers [such as hyaluronic acid (HA) and polycaprolactone (PCL)], and inorganic materials (such as calcium phosphate). With continuous degradation of the encapsulation materials, the bioactive agents are gradually released into the target area to achieve sustained release (Figure 3). To locally release microRNA-21, Wang et al. (2015) used CS and HA to encapsulate miRNA-21 to fabricate the CS/HA/miRNA-21 nanoparticles, which were then crosslinked with gelatin and loaded onto the implant surface, ultimately promoting the expression of the osteogenic gene. PCL and poly(lactic-co-glycolic) acid can also be used as encapsulation materials of bioactive agents to achieve local controlled release (Littuma et al., 2020). Biomimetic coprecipitation is a method of encapsulating bioactive agents with inorganic materials. With absorption of the hard coating, the bioactive agents are released gradually.

\section{CONCLUSION AND PERSPECTIVE}

Herein we have summarized the bioactive agents available to improve osseointegration in osteoporotic patients and the methods of controlled release of bioactive agents. High bioavailability and low toxicity in tissues outside the target make biomodification of implants suitable for achieving local osseointegration. Concerns about biosafety, however, limit the popularization of bioactive modification of implants, which is the direction of follow-up research. Implant osseointegration also

\section{REFERENCES}

Alenezi, A., Galli, S., Atefyekta, S., Andersson, M., and Wennerberg, A. (2019). Osseointegration effects of local release of strontium ranelate from implant surfaces in rats. J. Mater. Sci. Mater. Med. 30:116. doi: 10.1007/s10856-0196314-y

Apostu, D., Lucaciu, O., Lucaciu, G. D., Crisan, B., Crisan, L., Baciut, M., et al. (2017). Systemic drugs that influence titanium implant osseointegration. Drug Metab. Rev. 49, 92-104. doi: 10.1080/03602532.2016.1277737

Asri, R. I. M., Harun, W. S. W., Samykano, M., Lah, N. A. C., Ghani, S. A. C., Tarlochan, F., et al. (2017). Corrosion and surface modification on biocompatible metals: a review. Mater. Sci. Eng. C Mater. Biol. Appl. 77, 1261-1274. doi: 10.1016/j.msec.2017.04.102

Beck, F., Hartmann, E. S., Koehler, M. I., Redeker, J. I., Schluessel, S., Schmitt, B., et al. (2019). Immobilization of denosumab on titanium affects osteoclastogenesis of human peripheral blood monocytes. Int. J. Mol. Sci. 20:1002. doi: 10.3390/ijms20051002

Buser, D., Sennerby, L., and De Bruyn, H. (2017). Modern implant dentistry based on osseointegration: 50 years of progress, current trends and open questions. Periodontol 2000 73, 7-21. doi: 10.1111/prd. 12185

Chen, J., and Zou, X. (2019). Self-assemble peptide biomaterials and their biomedical applications. Bioact. Mater. 4, 120-131. doi: 10.1016/j.bioactmat. 2019.01.002 must be accompanied by angiogenesis and ingrowth, and it is an inflammatory regulatory process, which is initially mediated by M1 phenotype macrophages and subsequently by M2. In addition, bacterial colonization and formation of plaque biofilms on the surface of implants may cause peri-implantitis, ultimately leading to failure of the implant. Therefore, angiogenesis and ingrowth, regulation of the inflammatory response, and inhibition of biofilm formation also should be considered in future studies.

\section{AUTHOR CONTRIBUTIONS}

CZ and TZ wrote the original draft equally. TG helped to prepare the manuscript. XW gave conceptualization on this manuscript. KL and PW led the conceptualization and project administration, and supervised the writing and editing of the manuscript. All authors contributed to the article and approved the submitted version.

\section{FUNDING}

The National Natural Science Foundation of China (81871490 and 81700954), Program of Shanghai Academic/Technology Research Leader (19XD1434500), and Two-Hundred Talent (20191819) supported this study.

\section{ACKNOWLEDGMENTS}

We thank LetPub (www.letpub.com) for its linguistic assistance during the preparation of this manuscript. We also thank Xudong Wang of Shanghai Ninth People's Hospital, China, for feedback on drafts of this article.

Eguia, A., Bagan-Debon, L., and Cardona, F. (2020). Review and update on drugs related to the development of osteonecrosis of the jaw. Med. Oral. Patol. Oral. Cir. Bucal. 25, e71-e83. doi: 10.4317/medoral.23191

Gennari, L., Merlotti, D., Falchetti, A., Eller Vainicher, C., Cosso, R., and Chiodini, I. (2020). Emerging therapeutic targets for osteoporosis. Expert. Opin. Ther. Targets 24, 115-130. doi: 10.1080/14728222.2020.1726889

Hamlekhan, A., Sinha-Ray, S., Takoudis, C., Mathew, M. T., Sukotjo, C., Yarin, A. L., et al. (2015). Fabrication of drug eluting implants: study of drug release mechanism from titanium dioxide nanotubes. J. Phys. D Appl. Phys. 48, 275401. doi: 10.1088/0022-3727/48/27/275401

Ion, R., Necula, M. G., Mazare, A., Mitran, V., Neacsu, P., Schmuki, P., et al. (2020). Drug delivery systems based on titania nanotubes and active agents for enhanced osseointegration of bone implants. Curr. Med. Chem. 27, 854-902. doi: 10.2174/0929867326666190726123229

Jin, A., Wang, Y., Lin, K., and Jiang, L. (2020). Nanoparticles modified by polydopamine: working as "drug" carriers. Bioact Mater. 5, 522-541. doi: 10. 1016/j.bioactmat.2020.04.003

Jurczak, P., Witkowska, J., Rodziewicz-Motowidło, S., and Lach, S. (2020). Proteins, peptides and peptidomimetics as active agents in implant surface functionalization. Adv. Colloid. Interface Sci. 276:102083. doi: 10.1016/j.cis. 2019.102083

Lai, M., Yan, X., and Jin, Z. (2018). The response of bone cells to titanium surfaces modified by simvastatin-loaded multilayered films. J. Biomater. Sci. Polym. Ed. 29, 1895-1908. doi: 10.1080/09205063.2018.1517483 
Lin, G., Zhou, C., Lin, M., Xu, A., and He, F. (2019). Strontium-incorporated titanium implant surface treated by hydrothermal reactions promotes early bone osseointegration in osteoporotic rabbits. Clin. Oral. Implants Res. 30, 777-790. doi: 10.1111/clr.13460

Lin, K., Xia, L., Li, H., Jiang, X., Pan, H., Xu, Y., et al. (2013). Enhanced osteoporotic bone regeneration by strontium-substituted calcium silicate bioactive ceramics. Biomaterials 34, 10028-10042. doi: 10.1016/j.biomaterials.2013.09.056

Littuma, G. J. S., Sordi, M. B., Borges Curtarelli, R., Aragonês, Á, da Cruz, A. C. C., and Magini, R. S. (2020). Titanium coated with poly(lactic-co-glycolic) acid incorporating simvastatin: biofunctionalization of dental prosthetic abutments. J. Periodontal. Res. 55, 116-124. doi: 10.1111/jre.12695

Liu, J., Pathak, J. L., Hu, X., Jin, Y., Wu, Z., Al-Baadani, M. A., et al. (2018). Sustained release of zoledronic acid from mesoporous TiO2-Layered implant enhances implant osseointegration in osteoporotic condition. J. Biomed. Nanotechnol. 14, 1965-1978. doi: 10.1166/jbn.2018.2635

Liu, L., Wang, X., Zhou, Y., Cai, M., Lin, K., Fang, B., et al. (2020). The synergistic promotion of osseointegration by nanostructure design and silicon substitution of hydroxyapatite coatings in a diabetic model. J. Mater. Chem. B 8, 2754-2767. doi: $10.1039 / \mathrm{c} 9$ tb02882j

Liu, L., Yu, F., Li, L., Zhou, L., Zhou, T., Xu, Y., et al. (2021). Bone marrow stromal cells stimulated by strontium-substituted calcium silicate ceramics: release of exosomal miR-146a regulates osteogenesis and angiogenesis. Acta Biomater. 119, 444-457. doi: 10.1016/j.actbio.2020.10.038

Long, E. G., Buluk, M., Gallagher, M. B., Schneider, J. M., and Brown, J. L. (2019). Human mesenchymal stem cell morphology, migration, and differentiation on micro and nano-textured titanium. Bioact. Mater. 4, 249-255. doi: 10.1016/j. bioactmat.2019.08.001

Ma, Q., Jiang, N., Liang, S., Chen, F., Fang, L., Wang, X., et al. (2020). Functionalization of a clustered $\mathrm{TiO}(2)$ nanotubular surface with platelet derived growth factor-BB covalent modification enhances osteogenic differentiation of bone marrow mesenchymal stem cells. Biomaterials 230:119650. doi: 10.1016/j.biomaterials.2019.119650

Meng, H. W., Chien, E. Y., and Chien, H. H. (2016). Dental implant bioactive surface modifications and their effects on osseointegration: a review. Biomark Res. 4:24. doi: 10.1186/s40364-016-0078- z

Mu, C., Hu, Y., Huang, L., Shen, X., Li, M., Li, L., et al. (2018). Sustained raloxifene release from hyaluronan-alendronate-functionalized titanium nanotube arrays capable of enhancing osseointegration in osteoporotic rabbits. Mater. Sci. Eng. C Mater. Biol. Appl. 82, 345-353. doi: 10.1016/j.msec.2017.08.056

Shi, Q., Qian, Z., Liu, D., and Liu, H. (2017). Surface modification of dental titanium implant by layer-by-layer electrostatic self-assembly. Front. Physiol. 8:574. doi: 10.3389/fphys.2017.00574

Souza, J. C. M., Sordi, M. B., Kanazawa, M., Ravindran, S., Henriques, B., Silva, F. S., et al. (2019). Nano-scale modification of titanium implant surfaces to enhance osseointegration. Acta Biomater. 94, 112-131. doi: 10.1016/j.actbio.2019.05.045

Stewart, C., Akhavan, B., Wise, S. G., and Bilek, M. M. M. (2019). A review of biomimetic surface functionalization for bone-integrating orthopedic implants: mechanisms, current approaches, and future directions. Prog. Mater. Sci. 106:100588. doi: 10.1016/j.pmatsci.2019.100588

Takanche, J. S., Kim, J. E., Kim, J. S., Lee, M. H., Jeon, J. G., Park, I. S., et al. (2018). Chitosan-gold nanoparticles mediated gene delivery of c-myb facilitates osseointegration of dental implants in ovariectomized rat. Artif Cells Nanomed. Biotechnol. 46, S807-S817. doi: 10.1080/21691401.2018.151 3940

Tang, J., Yan, D., Chen, L., Shen, Z., Wang, B., Weng, S., et al. (2020). Enhancement of local bone formation on titanium implants in osteoporotic rats by biomimetic multilayered structures containing parathyroid hormone (PTH)-related protein. Biomed. Mater. 15:045011. doi: 10.1088/1748-605X/ ab7b3d
Teng, F. Y., Tai, I. C., Ho, M. L., Wang, J. W., Weng, L. W., Wang, Y. J., et al. (2019). Controlled release of BMP-2 from titanium with electrodeposition modification enhancing critical size bone formation. Mater. Sci. Eng. C Mater. Biol. Appl. 105:109879. doi: 10.1016/j.msec.2019.109879

Wang, H., Zhang, X., Wang, H., Zhang, J., Li, J., Ruan, C., et al. (2018). Enhancing the osteogenic differentiation and rapid osseointegration of $3 \mathrm{~d}$ printed ti6al4v implants via nano-topographic modification. J. Biomed. Nanotechnol. 14, 707715. doi: 10.1166/jbn.2018.2551

Wang, H., Liu, J., Wang, C., Shen, S. G., Wang, X., and Lin, K. (2020). The synergistic effect of $3 \mathrm{D}$-printed microscale roughness surface and nanoscale feature on enhancing osteogenic differentiation and rapid osseointegration. J. Mater. Sci. Technol. (in press). doi: 10.1016/j.jmst.2019.12.030

Wang, J., He, X. T., Xu, X. Y., Yin, Y., Li, X., Bi, C. S., et al. (2019). Surface modification via plasmid-mediated pLAMA3-CM gene transfection promotes the attachment of gingival epithelial cells to titanium sheets in vitro and improves biological sealing at the transmucosal sites of titanium implants in vivo. J. Mater. Chem. B 7, 7415-7427. doi: 10.1039/c9tb01715a

Wang, Z., Wu, G., Feng, Z., Bai, S., Dong, Y., Wu, G., et al. (2015). Microarc-oxidized titanium surfaces functionalized with microRNA-21-loaded chitosan/hyaluronic acid nanoparticles promote the osteogenic differentiation of human bone marrow mesenchymal stem cells. Int. J. Nanomed. 10, 66756687. doi: 10.2147/IJN.S94689

Xia, L., Xie, Y., Fang, B., Wang, X., and Lin, K. (2018). In situ modulation of crystallinity and nano-structures to enhance the stability and osseointegration of hydroxyapatite coatings on Ti-6Al-4V implants. Chem. Eng. J. 347, 711-720. doi: 10.1016/j.cej.2018.04.045

Xue, T., Attarilar, S., Liu, S., Liu, J., Song, X., Li, L., et al. (2020). Surface modification techniques of titanium and its alloys to functionally optimize their biomedical properties: thematic review. Front. Bioeng. Biotechnol. 8:603072. doi: $10.3389 /$ fbioe. 2020.603072

Yu, J., Xu, L., Li, K., Xie, N., Xi, Y., Wang, Y., et al. (2017). Zinc-modified calcium silicate coatings promote osteogenic differentiation through TGF-beta/Smad pathway and osseointegration in osteopenic rabbits. Sci. Rep. 7:3440. doi: 10. 1038/s41598-017-03661-3665

Zavan, B., Ferroni, L., Gardin, C., Sivolella, S., Piattelli, A., and Mijiritsky, E. (2017). Release of VEGF from dental implant improves osteogenetic process: preliminary in vitro tests. Materials (Basel) 10:1052. doi: 10.3390/ma10091052

Zhang, J., Liu, J., Wang, C., Chen, F., Wang, X., and Lin, K. (2020). A comparative study of the osteogenic performance between the hierarchical micro/submicrotextured 3D-printed Ti6Al4V surface and the SLA surface. Bioact. Mater. 5, 9-16. doi: 10.1016/j.bioactmat.2019.12.008

Zhao, C., Lin, K., and Wang, X. (2020). Maintenance and modulation of stem cells stemness based on biomaterial designing via chemical and physical signals. Appl. Mater. Today 19:100614.

Zhao, Q., Yi, L., Jiang, L., Ma, Y., Lin, H., and Dong, J. (2019). Osteogenic activity and antibacterial ability on titanium surfaces modified with magnesium-doped titanium dioxide coating. Nanomedicine (Lond) 14, 1109-1133. doi: 10.2217/ nnm-2018-2413

Conflict of Interest: The authors declare that the research was conducted in the absence of any commercial or financial relationships that could be construed as a potential conflict of interest.

Copyright (c) 2021 Zhang, Zhang, Geng, Wang, Lin and Wang. This is an open-access article distributed under the terms of the Creative Commons Attribution License (CC BY). The use, distribution or reproduction in other forums is permitted, provided the original author(s) and the copyright owner(s) are credited and that the original publication in this journal is cited, in accordance with accepted academic practice. No use, distribution or reproduction is permitted which does not comply with these terms. 